\title{
LAND SUITABILITY ASSESSMENT FOR DISASTER-PRONE VILLAGES RELOCATION
}

\author{
H.-L. LIN \\ Department of Urban Planning, National Cheng-Kung University, Taiwan.
}

\begin{abstract}
Lessons learnt from 921 Jiji Earthquake's recovery, suitable relocation sites are critical to land planning institutions to implement disaster recovery plans and to propose creative solutions to address unforeseen difficulties quickly. It is the time matter in disaster recovery and reconstruction management that the longer it takes, the higher uncertainty it becomes, resulting in lengthy, complex relocation processes. A virtual relocation land reserve system is suggested that site alternatives for relocation are pre-prepared through a land suitability assessment. To this end, the concept and advanced GIS techniques of disaster risk management were applied to disaster-prone village identification and the land suitability analysis for safety relocation land assessment. The article first created disaster risk maps of Taiwan to see the distribution of the disaster-prone village, in which disaster hazardousness and vulnerability were discussed. The relocation projects of $921 \mathrm{Jiji}$ Earthquake were also reviewed and concluded that there are six key factors for successful relocations, which are land regulations, location safety, financial feasibility, social and cultural connections, economic vitality, and awareness and willingness of community. The factors were further developed into criteria and were used in the land suitability analysis of relocation site assessment for disaster-prone areas.
\end{abstract}

Keywords: Disaster recovery, land suitability analysis, relocation, risk analysis, risk map, vulnerability.

\section{INTRODUCTION}

Experiences from 1999 Jiji Earthquake (also known as 921 Earthquake) [1,2] in Taiwan and recent devastating disasters worldwide have provided us with an important lesson that time as a factor in disaster recovery and reconstruction management is critical. Rehabilitation, resettlement, and relocation typically merge into a lengthy, complex process. Well-designed strategies and standard operating procedures (SOP) for relocation projects with commonly accepted, readily accessible relocation land assessments can significantly simplify and shorten the process. The need for such assessments for disaster-prone areas in Taiwan motivated the three-year (20082011) project Approaches and Criteria to Designate Relocation Sites (ACDRS). The project adopted the concept and advanced techniques in disaster risk management and GIS (geographic information system) land suitability analysis for assessing land safety. Its goals include the development of land use strategies that meet disaster risk reduction and mitigation requirements.

The project started with an initial risk analysis framework for assessing the safety of lands in disaster-prone areas within Taiwan and their suitability for relocation purposes. The framework was then enhanced on the basis of a broad spectrum of data, including data on debris flows, landslide and flood disasters, as well as the National Land-use Census data from 2009. Debris flow, landslide, and flood risk maps of disaster-prone areas in Taiwan were generated based on their impacts on the vulnerability of population, industry, public facility, and public utility in the areas. The risk maps, presented later in the article, enabled the identification of villages in Taiwan with high risk of debris flows, landslides, and floods. One hundred of the high-risk villages thus identified were surveyed to collect more detailed information at the community scale for designation of safe relocation site purposes.

In addition to risk maps, the results of the project include relocation land assessments for the selected high-risk villages. These results were integrated into a Virtual Relocation Land 
Reserve System (VRLRS). This information system contains official land assessments of candidate relocation sites as well as administrative SOPs to get the information on the relocation sites. The system can provide displaced disaster victims with rapid responses to requests for rehabilitation, resettlement, and relocation lands and geo-information.

Following this introduction, the remainder of the article is organized as follows. Section 2 discusses factors of relocation considered by the project. Section 3 presents an overview of VRLRS, including the major steps of its construction process. The risk maps and relocation land assessments for selected high-risk villages produced by the project are presented in Sections 4 and 5, respectively, together with the methods and processes of risk mapping and land assessment. Section 6 summarizes the article.

\section{FACTORS OF RELOCATION}

The primary objective of post-disaster relocation $[3,4]$ of a community can range from merely the restoration of the community and livelihood of people in it back to its former state to the encouragement of dramatic improvements of the living standard and social conditions of the community. Our land assessment and risk analysis methods take into account of the primary objective of each relocation project as well as six factors that can affect the chance of success of the relocation project. The factors are categorized into two groups presented in sections 2.1 and 2.2. The first group of factors, containing Regulations, Location Safety, and Financial Feasibility, can directly affect the planning and implementation of the relocation project. The second group of factors contains Social and Cultural Connections, Economic Vitality, and Victims' Awareness and Willingness. These factors affect the well-being of disaster victims to be relocated.

\subsection{Regulations, location safety, and financial feasibility}

In most parts of the world, relocation projects typically are required to adhere to numerous regulations governing land use and constructions. These regulations and institutional procedures of authorities overseeing relocation affairs directly affect the time and efforts required for planning, implementation, and administration of every post-disaster relocation project. For example, in Taiwan, lands for relocation must be in the 'Residence' category. To get approval for changing the zoning category of a chosen land from non-residence category to residence category, a relocation project must deal with rigid regulations and lengthy procedures, and risk the chance of failure to get the approval. If the chosen relocation site were on a hillside or in environmentally sensitive area, the project would be required to submit environment impact statements or evaluations in order to get construction licences. By providing GIS information on ecological and culture landscapes, disaster sensitivity maps, etc., our VRLRS aims to support the identification of lands unsuitable for relocation for regulatory and procedure reasons.

Location safety is another important consideration. Our land assessment and risk analysis methods start by identifying low disaster-risk regions from maps of potential debris flows, flooding depths, and landslide areas, which are provided by the Soil and Water Conservation Bureau, Water Conservation Agency, and Central Geological Survey, respectively [5-7]. The safety of candidate relocation lands were then verified within the low-risk regions by field surveys.

Relocation projects must be financially feasible. Published statistics showed that more than $50 \%$ of relocation-project failures resulted from financial issues. For this reason, cost of lands for relocation must be taken into account. In Taiwan, this means placing high priority on land owned by governments or state-owned enterprises. 
2.2 Social and cultural connections, economic vitality, and victims' awareness and willingness

Social and cultural connections are important for surviving disaster victims. This factor is particularly important for indigenous peoples. Taiwan's indigenous groups have a long history of living in mountainous areas, which attribute to their identities and living styles. Past experiences show that it was difficult to preserve indigenous culture when the sites of relocation were far away from the original villages. For this reason, our relocation land assessment methods assume that the closer the relocation site is to the original village, the higher the chance for social and cultural connections to be maintained or rebuilt. The methods also take into account the accessibility to original villages.

Disaster victims usually prefer reconstruction at the same place rather than relocation. It is more acceptable for them if the relocation sites are relatively advantageous in providing them with their former types of employment. Our methods take into account this factor by giving higher weights to lands closer to agricultures and industries and to locations nearer to homelands of the victims.

Finally, land ownership, family, and neighbor ties are among reasons for victims' desire to remain, even when their homelands are badly damaged and reconstruction is more costly than relocation. The information provided by our methods and VRLRS aims to support the challenging tasks of raising the awareness and willingness of victims to relocate.

\section{VIRTUAL RELOCATION LAND RESERVE SYSTEM}

As stated earlier, VRLRS provides relocation project planners and administrators and disaster victims with ready access to data and information on assessments of relocation lands and regulations governing land development and constructions. It also provides SOPs for planners and administrators so that they can easily follow relocation development guidelines, regulations, and best practices. In particular, VRLRS can help them launch relocation projects for villages victimized by debris flow, landslide, and flood disasters in the shortest time. The GIS databases, web services, and tools in VRLRS were prepared during disaster-free period, and are continuously updated and maintained. Thus the databases can also contribute to efforts in disaster prevention and risk reduction for disaster-prone communities.

Compared with regular development projects, disaster relocation-reconstruction projects are much more complicated and are under much higher time pressure. For this reason, it is important to establish a simplified administration procedure to coordinate the dialogues between various authorities in community, local, and central government levels. This is another kind of support provided by VRLRS. The development guidelines and regulations suggested by VRLRS are in checklist forms. The forms also provide links to information needed to get development permissions, including environment safety evaluation reports, environment impact statement/ analysis, water and soil conservation plans, engineering geological surveys, etc.

In the process of constructing VRLRS, ACDRS project exploited lessons and experiences gained from 2009 Morakot Typhoon and 921 Earthquake homeland redevelopment [8]. The construction process consists of the following four stages:

1. Identification and designation: In this stage, official GIS disaster information databases were used to generate disaster risk maps of Taiwan. Villages prone to debris flows, landslides, and floods were identified based on information provided by the maps. The risk index of each identified village was then calculated. The results are risk distribution maps for these types of disasters in Taiwan. The next section provides further details. 
2. Pre-assessment: The goal of this stage is to seek suitable relocation lands for each specified high disaster-risk village based on available GIS map resources. The assessment of land location follows the principle of finding 'the nearest suitable safe place'. Hence the priorities of relocation sites are lands within the village, the township, and then the nearest suitable lands, in descending order of priorities.

3. Vulnerability and safety field survey: A field survey conducted in this stage is an integral part of land assessment and risk analysis methods. The goal is to confirm the accuracy of pre-assessment and to designate the boundary of each relocation site. Major elements of fieldwork are local interviews and field research as well as surveys of disaster types, current vulnerabilities of the location, and the current settlement status on record for each selected high-risk village. This work is a part of the validation process. At the end of the survey, location facts about each relocation site are based on results from the field survey, not second-hand GIS data.

4. Guidelines and regulations for relocation development: Based on the results of field survey on land safety and suitability assessments, guidelines and regulations of each relocation site were developed according to the legislation entitled or required. Format and forms of zoning maps and legislation tables were designed accordingly.

Before moving on, it should be noted that field survey is performed also for the sake of reducing data uncertainties. The risk maps based on land assessment may differ from the current situation because of uncertainty in the data used to generate the maps [9]. There are three kinds of uncertainties: The first is uncertainty in time and space, including uncertainties in disaster location, time, scope of influence, etc. This kind of uncertainty arises from the fact that every disaster is dynamic, whereas the relevant GIS information on the disaster is based on data captured at specific times. The second kind of uncertainty is in scale. For different types of disaster and the spatial extent of the planning process, the scale of existing map may be different. Due to limitations in accuracy and unit size, a map may not provide sufficiently accurate information required in the development stage. The third kind of uncertainty is in subjective and objective viewpoints on the information required for disaster prevention decision making. An example of this kind of uncertainty is the difference between analysed results and local opinions. A relocation site suggested by risk assessment may not meet some requirement deemed important by disaster victims. Our current work has shown that using data and information acquired first hand by researchers from interviews and meetings with people in each assessed area has proved to be an effective means to reduce data uncertainty [10].

\section{GENERATION OF DISASTER RISK MAP}

Risk identification is the preliminary analysis for disaster mitigation strategy of relocation projects. The Office of United Nations Disaster Relief Co-ordinator (UNDRO) [11] defined the term 'risk' as the expected losses from a given hazard to a given risk element. The definition is usually presented by the conceptual equation model below:

$$
\text { Risk = Hazard } \times \text { Vulnerability }
$$

The hazard-specific risk combines the probabilities of the level of a specific hazard and its impact to population's vulnerability in terms of lives, properties or economics lost, etc.

Figure 1 illustrates the framework of overlay mapping used by this research to support the risk analysis to identify hazard-prone villages. Overlay mapping is widely used for the generation of disaster risk map. The method addresses hazard's risk spatial characteristics 


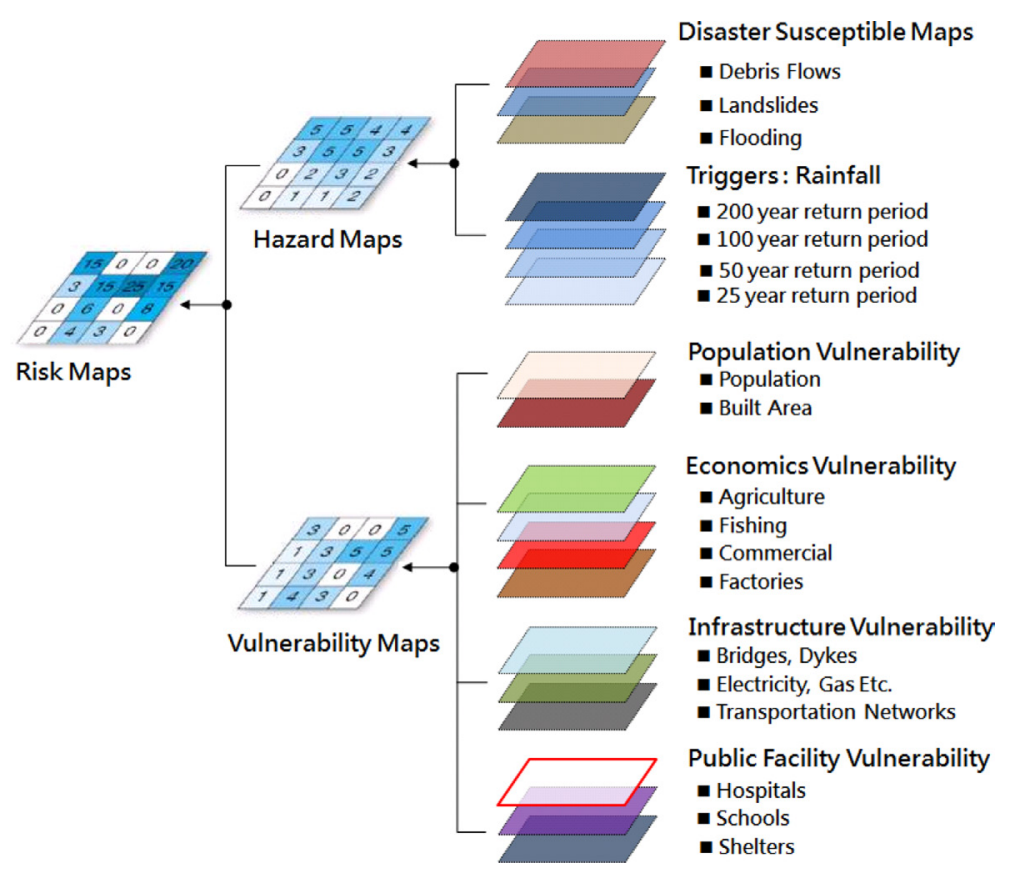

Figure 1: Overlay approach to generating disaster risk maps.

explicitly, which is important for spatial planning strategies of disaster mitigation. As Fig. 1 shows, hazard and risk maps of debris flow, landslides, and flood were derived from their own disaster susceptibility maps, overlaid with vulnerability maps of population, land use, public utilities, and public facilities (including transportation network) [12].

Within the framework, susceptibility maps of debris flow, landslide, and floods, obtained from government authorities of Soil and Water Conservation Bureau, Central Geological Survey, and Water Resources Agency, respectively. These maps were first overlaid with the recorded level of rainfall in a given period of time to obtain three hazard maps of Taiwan. The susceptibility maps contain the topographical information of elevation, slope, aspect, etc. The hazard scores, measurement of hazardousness, were calculated by multiplying the susceptibility scores and the recorded rainfall levels. The hazard map is spatial representation of hazard scores that indicates the dangerousness of the study area, i.e. the potential impact to the region that the hazard associated with the environment factors of exposures will cause. The hazard map scores are then transformed into a ranking-based representation for reasons and in ways described in subsequent paragraphs. Meanwhile, the built environment vulnerability of population, economics, and infrastructure are collected and re-classified into 5-level ranking score of vulnerability. Finally, the disaster risk maps are generated by taking products of the corresponding values given by hazard maps and vulnerability maps.

Figure 2 illustrated three thematic maps of debris flow risk analysis. Figure 2a presented the location of 1,503 potential debris flow torrents over the island of Taiwan. The assessment of the debris flow hazardousness in the scenario of 25-year return period rainfall showed in Fig. 2b. Villages' risk scores were a map overlay operation with the maps of the debris flow hazardousness and villages' vulnerability in ArcGIS software. Figure $2 \mathrm{c}$ demonstrated the assessment of the debris flow risk of Taiwan. 


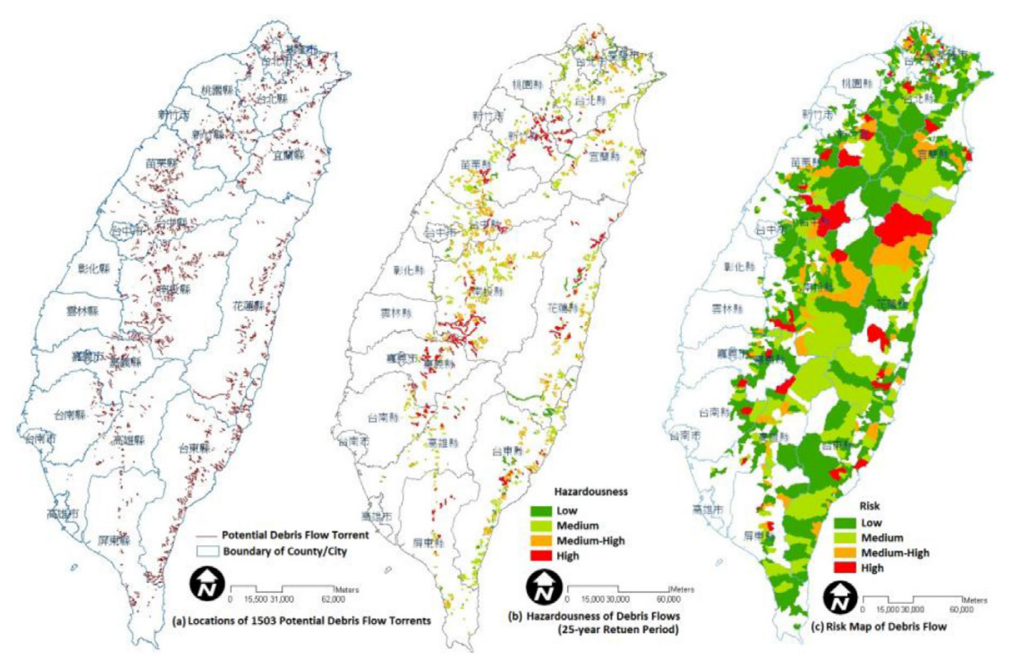

Figure 2: Debris flow risk analyses.

The conventional approach of generating risk map is often heuristic and subjective as assignment of susceptibility level to risk can vary from one type of hazard map to another. In addition, the differences in ranges of values between hazard maps and vulnerability maps influence the final scores used in the disaster risk maps, which may give a false representation of the overall level of risk for a region.

This problem was removed by standardizing the scores of hazard, and vulnerability maps prior to obtaining the product of their scores in order to minimize the differences in value ranges between maps: Risk levels are separated into five categories: no risk, low, medium, medium-to-high, and high. The categories are defined by ranges of risk score below the mean, between the mean and 1.0 standard deviation, between 1.0 and 2.0 standard deviations, and between 2.0 and 3.0 standard deviations, and above 3.0 standard deviations, respectively. An overview of the distribution of data values may reveal that additional characteristics and classification of risk category can be tailored to a specific region. Furthermore, standardization of scores enables a highly statistical approach to risk classification.

Figure 3 presented the debris flow risk scores of 963 villages of Taiwan by graphs of histogram and line-chart. The $x$-axis indicates the risk Z-score and $y$-axis is the number of villages in the risk $Z$-score range of 0.2 increments. The histogram shows a positive skew that the data have long tails on the right side of mean. High-risk Z-scores indicate relatively serious damages that local debris flows is likely to cause to local villages. A village is said to be hazard prone when its risk Z-score is higher than 2.0 after a cross-check with the disaster historical records.

The top five debris flow disaster-prone counties of Taiwan are listed in Table 1. Number of villages in each risk category and their coverage area in square kilometres (denoted by $\mathrm{km}^{2}$ ) are listed in each column. The epicentre of the 921 Jiji Earthquake was in NanTou county. The county contains the largest number of villages in the medium-to-high risk categories in the region. The two counties adjacent to NanTou are HuaLien and ChiaYi. They are the second and third highest risk disaster-prone regions, respectively, followed by HsinChu and TaiTung, the two counties located further away from the epicentre. Correlation between disaster-prone zone and mountainous terrain is apparent as parts of Taiwan's mountain ranges 


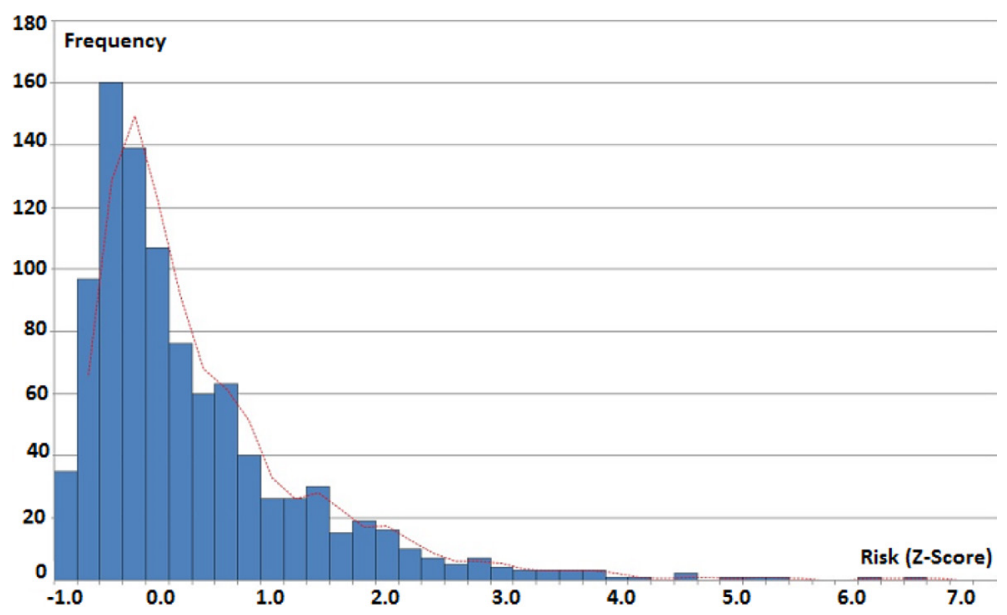

Figure 3: Histogram of the debris flow risk scores for 963 villages in Taiwan.

Table 1: Debris flow risk assessment of the top five counties of Taiwan.

\begin{tabular}{lcrrrrrrrrr}
\hline & \multicolumn{2}{c}{ Low } & \multicolumn{9}{c}{ Medium } & \multicolumn{4}{c}{ Medium-to-high } & \multicolumn{2}{c}{ High } & Total area \\
\cline { 2 - 10 } County & Villages & $\mathrm{km}^{2}$ & Villages & $\mathrm{km}^{2}$ & Villages & $\mathrm{km}^{2}$ & Villages & $\mathrm{km}^{2}$ & $\left(\mathrm{~km}^{2}\right)$ \\
\hline NanTou & 67 & 0.87 & 27 & 137.04 & 13 & 185.02 & 3 & 109.75 & $4,099.73$ \\
HuaLien & 45 & 66.39 & 21 & 81.86 & 6 & 106.01 & 5 & 85.44 & $4,593.46$ \\
ChiaYi & 23 & 0.95 & 9 & 10.56 & 3 & 23.05 & 2 & 85.14 & $1,947.71$ \\
HsinChu & 28 & 4.32 & 7 & 11.48 & 5 & 36.69 & 3 & 68.15 & $1,405.73$ \\
TaiTung & 47 & 61.3 & 22 & 151.67 & 7 & 113.46 & 3 & 36.15 & $3,561.38$ \\
\hline
\end{tabular}

span these five counties. Other counties close to NanTou (e.g. TaiChung, ChangHu, and YunLin) do not contain as many at-risk villages according to the risk assessment. This fact indicates that larger the proportion of mountainous terrain that the village consists of, the closer it is to the disaster zone, thus the higher the risk level.

\section{LAND SUITABILITY ASSESSMENT FOR RELOCATION}

According to the result of our debris flow risk assessment, 44 villages out of 963 were placed in the high debris flow risk category. These 44 villages need to have relocation policies to guide their efforts in mitigating population vulnerability and reducing disaster risks. Suitability land assessment was carried out to locate suitable relocation sites for each of these villages. Section 2 discussed attributes of relocation sites that affect planning and implementation of relocation projects and well-being of surviving disaster victims. The section also mentioned that it is important to reduce uncertainties in data used to support the assessment. The remainder of this section describes the three steps in the procedure developed by ACDRS project for assessing suitability of candidate relocation lands. 


\subsection{Mapping environment insecure/sensitive areas}

The assessment procedure first takes into account planning and implementation concerns, by excluding locations in environmentally sensitive areas and disaster-prone areas. Three categories of maps were collected from corresponding authorities for this purpose: maps of development-restricted areas, potential disaster areas, and strategic reconstruction areas. The maps provide information on three levels of regulatory control for different land uses.

The map of each development-restricted area takes into account of 19 environmentally sensitive lands, related to ecosystem sensitivities, cultural landscape, nature resources, nature disasters, etc. The map for each selected village was enacted in the second overall review of Taiwan Regional Planning. The second category of maps has information on flooding-prone areas, debris flow warning areas, and geological landslide-prone areas. Being aware of the fact that global climate change may have caused an increase in the frequency and intensity of typhoons in Taiwan area, Council for Economic Planning proposed a Regional Reconstruction Master Plan, in which, developments within an area for conservation of ecological resources, landscape resources, and soil and water resources are further limited. Land located within those areas should be excluded from consideration for the alternative relocation sites. Figure 4 shows the result of overlay mapping of the three categories of maps, using Xiaolin village as an example.

\subsection{Mapping sites with socio-economic and livability advantages}

ACDRS project used four accessibility indices to take into account factors affecting the well-being of surviving disaster victims. The indices are settlement, industry, road network, and public facility accessibilities. Settlement accessibility assumes that the nearer a relocation site is to the victims' village, the higher the possibility for the social and cultural connection to be maintained or rebuilt. Industry accessibility addresses the importance that relocation sites have relatively high advantage in providing jobs: The closer the candidate relocation site is to lands used for agricultures, retail, and industries, the higher its industry

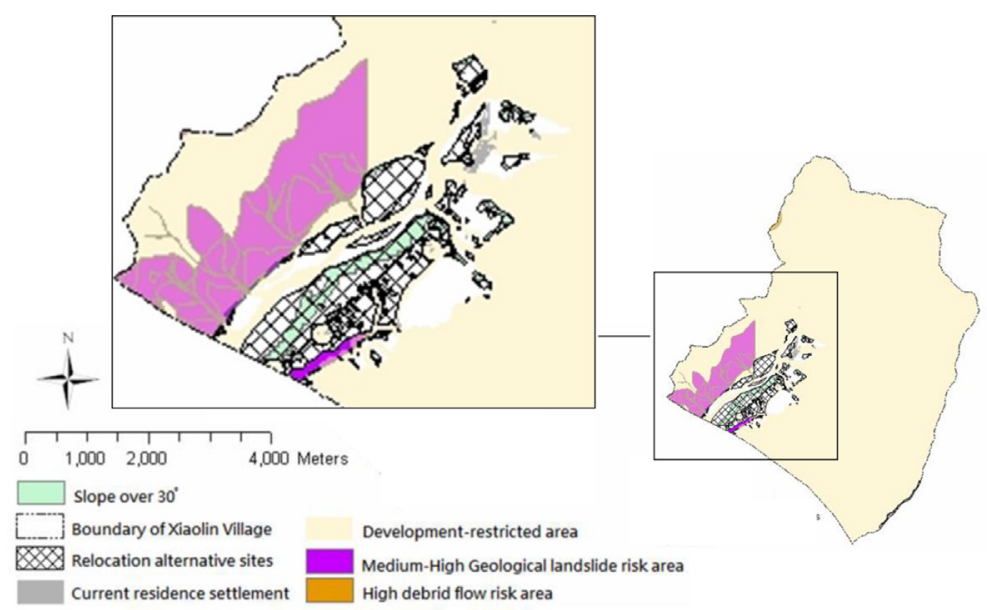

Figure 4: Maps of environmentally insecure and sensitive area of Xiaolin village. 

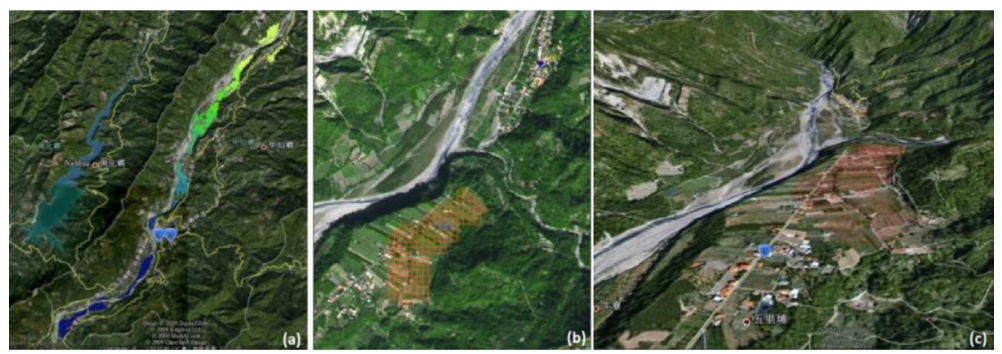

Figure 5: Accessibility and the relocation site over Google Earth.

accessibility index. Finally, sites with higher accessibility for road network and public facility are places with higher liveability and hence can provide relocation communities with better quality of life.

The maps shown in Fig. 5 are the result of accessibility assessment of Xiaolin village. In the figure, the accessibility map and the relocation sites are presented over Google Earth to show topographic perspective of safety concerns. Panel (a) is the accessibility map of a site that can be selected for relocation. Panel (b) shows the area selected for Xiaolin's relocation. Panel (c) provides a three-dimensional viewpoint of the selected area. For a high debris flow risk village like Xiaolin, table lands are more resilient to flood or debris flow disasters than valley lands. Compared with panel (b), panel (c) provides a much better viewpoint for checking this and other topographic factors over the area.

\subsection{Fixing the potential data uncertainty via field survey}

At the end of step 2 described above, suitable relocation sites have been identified. The goal of step 3 is to ensure that the maps generated so far by our assessment procedure are accurate and the relocation sites suggested are indeed suitable. Data uncertainty is a source of inaccuracies in maps and erroneous recommendations. Disaster risk assessment was first carried out at a national scale to target at-risk regions. The results obtained from overlaying maps of various scales, ranging from $1 / 100,000$ to $1 / 1,000$ are not always suitable for downstream analysis and lack the level of detail. The assimilation of available databases for purposes of generating different maps may introduce inaccuracy or incompatibility because the databases may not be up to date. Databases are often constructed by different departments for specific objectives, which may exclude data deemed non-relevant for their purposes. More importantly, views of disaster victims with regard to the selected relocation sites have yet to be considered, and their views influence the outcome of relocation greatly and must be taken into account.

The research carried out a field survey for each village to minimize data uncertainties and validate the accuracy of the assessment at local community scale, as well as taking into account victims' views. Field survey was designed to consider the type of disaster, the area affected, the vulnerability of built environment, and geological details $[13,14]$. This step offers a finer level of information toward deciding the relocation site.

The field survey constitutes interviews followed by land assessments. The interviews with the village representative and witnesses of past disasters aim to provide a narrative of how a past disaster event had occurred and the extent of the disaster from their points of views. Disaster zones and relocation sites suggested by our method and by the disaster victims are 


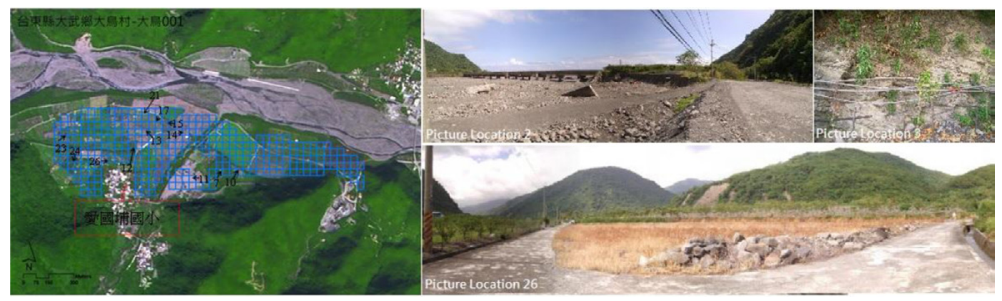

Figure 6: The geological map for field survey.

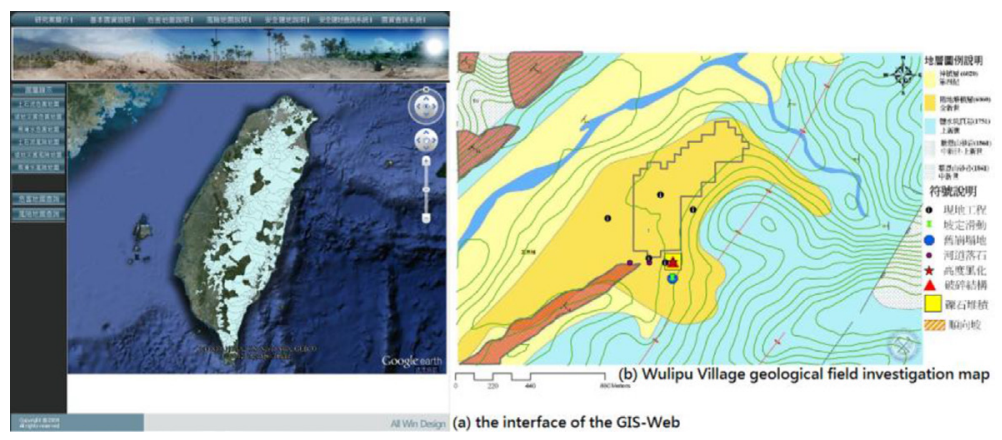

Figure 7: The Web-GIS platform of VRLRS.

then surveyed. The land assessment done in this step includes properties of soil, degree of vegetation, and geological marks, which can reveal hidden land characteristics. Figure 6 is an illustration of the geological map with the fact found during the field works. Finally, profiles of relocation sites are produced. The comprehensive assessment lists contain the detailed information on the location in terms of $X, Y$ coordinates, four accessibilities, land ownerships, land prices, land category, zoning regulation, and land use.

\subsection{Web-GIS database}

The presentation of the relocation land assessment information is also important $[15,16]$ in reducing the complexity of relocation projects for local authorities. ACDRS project applied Web-GIS technology to meet the requirement. Our Web-GIS platform, shown in Fig. 7, displays the assessment data, information, pictures, images, documents, etc.

Figure $7 \mathrm{a}$ is the interface of the platform by Google Earth that provides as a geo-reference map engine to display disaster information as maps. There are four types of hazard map information that can be queried, including debris flow, landslide, earthquake, and flood hazards. They provide data used for assessment: riskiness of villages, the hazardousness of villages, and the suggested relocation site for the top 100 riskiness villages. For the top 100 riskiness villages, the community-level detail of environment dangerousness and suitability assessment for relocation by field investigation is also provided. As an example, Fig. 7b shows the geological field investigation of $\mathrm{WuLiPu}$ village. In the map, fragile geological spots are labelled with various illustrations. Besides, the profile of alternative relocation sites' information such as land ownership, land prices, and land regulations, etc. can also be queried. 


\section{SUMMARY AND FUTURE WORK}

The VRLRS described in the previous sections is not just a land pre-assessment system. It is also a decision support system, as it provides relocation land assessments plus the SOP for relocation development projects. Such an SOP has four steps: (1) generate disaster risk maps and identify debris flow, landslides, flood-prone villages in high risk area(s); (2) carry out local land suitability analysis for relocation migration; (3) do field survey to improve accuracy in safety and liveability assessments from the village's perspectives; and (4) complete processes to conform to relocation guidelines and regulations. The system allows relocation projects to follow the principle of 'the nearest placement'. In this way, it enables the projects to put the social and cultural connectivity of affected disaster victims as one of the most important factors to consider in their choice of relocation site.

It is realized that from the viewpoint of disaster information uncertainty, disaster information in community level is particularly critical and vital for disaster-prone communities. Official disaster decision support systems typically lack community-level information. OpenISDM project [10] is seeking IT solutions of issues of open disaster information resources, by which, the disaster information by private/individuals sectors can contribute to disaster prevention and mitigation. Our future study of using open data will focus on the uncertainty reduction, including the uncertainty characteristic of open data, measurement of the uncertainty, uncertainty reduction, and efficiency assessment of the reduction processing.

\section{ACKNOWLEDGMENTS}

This work was supported by the research program 'Enhancing Innovation and Implementation of Disaster Reduction' funded by Urban and Rural Development Branch, Construction and Planning Agency of the Interior Ministry, Taiwan, in years 2008-2011, and Taiwan Academia Sinica project OpenISDM (Open Information Systems for Disaster Management) AS-102-SS-A04.

\section{REFERENCES}

[1] Jung, S.W., "Never again": narratives of suffering and memory of the 9/21 earthquake in Taiwan. Taiwan Journal of Anthropology, 7(1), pp. 35-65, 2009.

[2] 921 Earthquake Relief Foundation, available at www.taiwan921.lib.ntu.edu.tw

[3] Shieh, J.C., Chang, R., Tsai, P.H. \& Wang, C.K., Review of post-disaster village relocation policy in Taiwan. Journal of Housing Studies, 17(2), pp. 81-97, 2008.

[4] Chang, Y.-M., Tribe reconstruction planning (Chapter 5). A Study on the Reconstruction of San-Cha-Keng Atayal Aboriginal Tribe after 921 Earthquake Disaster, Master thesis, Department of Architecture: Tunghai University, pp. 5-1-5-20, 2007.

[5] Debris Flow Prevention Information System, Soil and Water Conservation Bureau, Council of Agriculture, Executive Yuan, available at 246.swcb.gov.tw

[6] Disaster Prevention Information Service Network, Water Resource Agency, Ministry of Economic Affairs, available at fhy2.wra.gov.tw/Pub_WebE_2012

[7] Interpretation of Geological Features Service System, Central Geological Survey, Ministry of Economic Affairs, available at gis.moeacgs.gov.tw/gwh/gsb97-1/sys8/index.cfm

[8] Morakot Post-Disaster Reconstruction Council, Executive Yuan, available at 88 flood. www.gov.tw/index.php

[9] Amendola, A., Management of change, disaster risk, and uncertainty: an overview. Journal of Natural Disaster Science, 26(2), pp. 55-61, 2004.

[10] OpenISDM, available at openisdm.iis.sinica.edu.tw 
[11] Office of the United Nations disaster relief co-ordinator. National Disasters and Vulnerability Analysis, 1979.

[12] Lin, H., An approach to access suitable lands for disaster mitigation. WIT Transactions on the Built Environment, 133, ISSN 1743-3509, 2013. doi: http://dx.doi.org/10.2495/ dman130201

[13] Taolin Digital Surveying Engineering Co. Ltd., The Primary and Revised Field Investigation and Security Assessment of Disaster Indigenous Tribe Villages by Typhoon Morakot, Council of Indigenous Peoples, Executive Yuan, 2009.

[14] Charng-Feng Engineering Consultants Ltd., Morakot Rehabilitation Housing Project - the site of Wu-Li-Pu Village, Jea-Shian, Kaohsiung, Kaohsiung City Government, 2009.

[15] Shieh, L.S., Su, W.R., Wu, C.R. \& Hwang, J.H., The field investigation for flooding and the development of flooding information management system. NGIS Quarterly, 61, pp. 27-40, 2007.

[16] Hsu, P.H., Wu, S.Y. \& Lin, F.T., Disaster management using GIS technology: a case study in Taiwan. Proceedings of the 26th Asia Conference on Remote Sensing, eds. Asian Association on Remote Sensing (AARS), Curran Associates, Inc., pp. 15101519, 2005. 